\title{
On Finsler geometry, MOND and diffeomorphic metrics to the Schwarzschild solution
}

\begin{abstract}
We revisit the construction of diffeomorphic but not isometric solutions to the Schwarzschild metric. The solutions relevant to Black Holes are those which require the introduction of non-trivial areal-radial functions that are characterized by the key property that the radial horizon's location is displaced continuously towards the singularity $(\mathrm{r}=0)$. In the limiting case scenario the location of the singularity and horizon merges and any infalling observer hits a null singularity at the very moment he/she crosses the horizon. This fact may have important consequences for the resolution of the firewall problem and the complementarity controversy in black holes. It is shown next how modified Newtonian dynamics (MOND) can be obtained from solutions to Finsler gravity, and which in turn, can also be modelled by metrics which are diffeomorphic but not isometric to the Schwarzschild metric. The key point now is that one will have to dispense with the asymptotic flatness condition, by choosing an areal radial function which is finite at $r=\infty$. Consequently, changing the boundary condition at $r=\infty$ leads to MONDian dynamics. We conclude with some discussions on the role of scale invariance and Born's Reciprocal Relativity Theory based on the existence of a maximal proper forces.
\end{abstract}

Keywords: general relativity, black holes, Finsler geometry, MOND, dark matter
Volume 2 Issue 6 - 2018

\author{
Carlos Castro Perelman \\ Center for Theoretical Studies of Physical Systems, Clark Atlanta \\ University, Georgia
}

\begin{abstract}
Correspondence: Carlos Castro Perelman, Center for Theoretical Studies of Physical Systems, Clark Atlanta University, Atlanta, GA. 303 I4, Ronin Institute, I 27 Haddon PI., NJ. 07043 ,
\end{abstract} USA,Email perelmanc@hotmail.com

Received: December 06, 2018 | Published: December 26, 2018
PACS: 04.60.-m, 04.65.+e, 11.15.-q, 11.30.Ly

Introduction: Diffeomorphic metrics to the Schwarzschild solution, firepoints and firewalls

The static spherically symmetric (SSS) vacuum solution of Einstein's field equations ${ }^{1}$ that we learned from the text books is actually the Hilbert form of the original Schwarzschild solution ${ }^{2}$

$$
(d s)^{2}=\left(1-\frac{2 G M}{r}\right)(d t)^{2}-\left(1-\frac{2 G M}{r}\right)^{-1}(d r)^{2}-r^{2}(d \Omega)^{2} .
$$

Birkoff's theorem states that all static spherically symmetric vacuum solutions to Einstein's equations are diffeomorphic to the Hilbert-Schwarzchild solution. There are an infinite number of metrics $^{3,4}$ which are diffeomorphic but not isometric to the Hilbert form of the Schwarzschild ${ }^{2}$ solution. In particular, given an areal radial function $\rho(r) \neq r$ (in $c=1$ units), the metric

$$
(d s)^{2}=\left(1-\frac{2 G M}{\rho(r)}\right)(d t)^{2}-\left(1-\frac{2 G M}{\rho(r)}\right)^{-1}(d \rho)^{2}-\rho^{2}(r)(d \Omega)^{2} .
$$

is diffeomorphic but not isometric to the Hilbert form of the Schwarzschild ${ }^{2}$ solution. $(d \rho)^{2}=(d \rho(r) / d r)^{2}(d r)^{2}$, and the solid angle infinitesimal element is $(d \Omega)^{2}=(d \phi)^{2}+\sin ^{2}(\phi)(d \theta)^{2}$. The surface area at each point $r$ is now given by $4 \pi(\rho(r))^{2}$ so that $\rho(r)$ plays the role of an effective radius and hence the name of "arealradial" function for $\rho(r)$.

The metric (1.2) is diffeomorphic but not isometric to the Hilbert form (1.1) of the Schwarzschild solution ${ }^{2}$ because the area elements $r^{2}(d \Omega)^{2} \neq \rho^{2}(r)(d \Omega)^{2}$ are not equal, except in the trivial case when $\rho(r)=r$. We have not relabelled the radial variable $r$ by giving it another "name" and calling it " $\rho$ ", because $\rho(r)$ is itself a function of $r$. Furthermore, one has not performed a naive change of radial coordinates $r \rightarrow \rho$ because the metric (1.2) is still given in terms of $r$. Therefore, the metric (1.2) is not the Schwarzschild metric rewritten in terms of new radial coordinates, but it is obtained after performing an active diffeomorphism via the mappings $r \rightarrow \rho(r)$. Under active diffeomorphism the points of the manifold are displaced (except for the fixed points) and must not be confused with passive diffeomorphisms that amount to mere coordinate transformations (like rotation, translation of the coordinate axes) leaving all the points fixed. Consequently, the metric (1.2) assumes the same values as the Schwarzschild metric (1.1) but at different values of $r$ (at different radial locations). This is the reason why the horizon can be actively displaced from $r=2 G M$ to the value $r=0^{+}$as we shall see below.

The boundary condition obeyed by the areal radial function $\rho(r)$ at the origin is $\rho(r=0)=0$ since the point mass location must reside at the center of spherical symmetry. At infinity, an asymptotically flat metric would require $\rho(r \rightarrow \infty) \sim r \rightarrow \infty$. In particular, the metric solutions (1.2) are invariant under the transformations $r \rightarrow-r ; M \rightarrow-M$ for the particular choice of areal radial functions obeying the condition $\rho(-r,-M)=-\rho(r, M)$. This allows us to extend the solutions to the $r<0$ region.

The metric (1.2) leads to modifications of the Newtonian potential, and for this reason, it is very relevant to modified Newtonian dynamics (MOND) as described in the next section. One recovers the Newtonian potential in the regime when $\rho(r) \simeq r$. In the next section we will show why modified Newtonian dynamics (MOND) $)^{5,6}$ associated with galaxies can be obtained from Finsler gravity, and which in turn, can be modeled by metrics (1.2) which are diffeomorphic but not isometric to the Hilbert-Schwarzschild metric. The key point is that one will have to dispense with the asymptotic flatness condition, and introduce an infrared-cut-off for the metric at $r=\infty$ in the form of $\rho(r=\infty)=\rho_{o}=$ finite. Therefore, changing the boundary condition at $r=\infty$ leads to MONDian dynamics. 
To model the scenario when the horizon merges precisely with the singularity one needs an area radial function defined at $r=0$ and at $r \neq 0$, as follows $\mathrm{s}^{3,4}$

$$
\rho(r=0)=0 ; \rho(r)=r\left(1-e^{-|r| 2 G|M|}\right)^{-1}, r \neq 0
$$

Under $r \rightarrow-r ; M \rightarrow-M$ one has that $\rho(r) \rightarrow-\rho(r)$ so one can ensure the invariance of the metric (1.2) under these transformations and extend the solutions to the $r<0$ region. $\rho(r)$ is antisymmetric $\rho(-r)=-\rho(r)$, and as such it must vanish at $r=0$.

Hence, we have in eq-(1.3) that $\rho\left(r=0^{+} ; M\right)=2 G M$, and $\rho\left(r=0^{-} ;-M\right)=-2 G M$, but $\rho(r=0)=0$ since a point mass must have zero area and zero volume. The horizon is located at $r_{h}=0^{+}$ and the singularity at $r=0$. There is a discontinuity of $\rho(r)$ at $r=0$ . The right $r=0^{+}$, and left $r=0^{-}$limits of $\rho(r)$ give respectively $\pm 2 G M$, while $\rho(r=0)=0$ which is the arithmetic mean of $2 G M$ and $-2 G M$. This is similar to the behaviour of the step function $\Theta(r)$ defined by $\Theta=1, r>0 ; \Theta=-1, r<0 ; \Theta=0, r=0$. $\Theta$ can be realized as $r /|r|$ which is an antisymmetric function, and as such, it must vanish at the origin, like the areal radial function in eq-(1.3).

Because a point mass is an infinitely compact source of infinite density, there is nothing wrong with the possibility of having a discontinuity of the metric at the location of the singularity $r=0$. Due to the boundary condition $\rho(r=0)=0$, there is a curvature tensor singularity and the Kretschmann invariant $R_{\mu v \rho \sigma} R^{\mu v \rho \sigma} \sim(2 G M)^{2} / \rho(r)^{6}$ diverges at $\rho(r=0)=0$. In this extreme case, when the location of the horizon merges with the singularity, there is a null-line singularity at $r=0$ and a null-surface at $r=0^{+}$. This may sound quite paradoxical but it is a consequence of the metric discontinuity at $r=0$, the location of the point mass (singularity). This key fact may have important consequences for the resolution of the firewall problem and the complementarity controversy in black holes. ${ }^{7,8}$ We may label the null-line singularity at $r=0$ as a firepoint, and the null-surface at $r=0^{+}$as a firewall. A recent discussion of the notion of a firepoint can be found $i^{8}$ where they propose the singularity itself as a "firepoint" capable to break the entanglement between the "in" and the "out" states created through the Hawking process.

When the areal-radial function $\rho(r)$ has the actual form in eq(1.3) there is no interior region beyond the horizon $r=0^{+}$, so that the metric (1.2) is truly static everywhere. The Fronsdal-KruskalSzekeres analytical continuation of the metric (1.1) inside the horizon is no longer static. Klinkhamer ${ }^{9}$ provided earlier on a regularization of the standard Schwarzschild solution with a curvature singularity at the center by removing the interior region of a ball and identifying the antipodal points on the boundary. The resulting four-dim manifold has now the topology $R \times \tilde{M}_{3}$ where $\tilde{M}_{3}$ is a nonsimply-connected manifold, which up to a point (the center), is homeomorphic to the 3 -dim real projective space $R P^{3}$. In our case, there is no need to remove the interior region by surgery. The discontinuity $\rho(r=0)=0 ; \rho\left(r=0^{+}\right)=2 G M$ of the areal-radial function amounts to a sort of "point-splitting" creating a void (hole) in spacetime, isolating and expunging the curvature singularity at the center from the remaining region of space-time. The topology of the region free of the singularity at the center is $\tilde{M}_{4}=R \times \tilde{M}_{3}$, where $\tilde{M}_{3}=R^{3}-\{0\}$ is the punctured 3-dim space.

\section{Finsler geometry, modified Newtonian dynamics and areal radial functions}

It has long been known that if one recurs to Newton's inversesquare law of gravity, the observed baryonic matter cannot provide enough force to attract the matter (stars) present in the outer edges of the galaxies. ${ }^{10,11}$ Postulating that galaxies are surrounded by massive, non-luminous dark matter is one of the most widely accepted proposals to solve the problem. No dark matter has been detected yet. An extensive overview with a vast number of references of the tests and problems of the standard model in Cosmology. ${ }^{12}$

Some models have been built as an alternative to the dark matter hypothesis. The main ideas are based on assuming that the Newtonian gravity or Newton's dynamics is invalid on galactic scales. In the MOND model (modified Newtonian dynamics) of Milgrom ${ }^{5,6}$ it assumes that the Newtonian dynamics does not hold on galactic scales. In Extended Theories of Gravity, like $f(R)$ gravity ${ }^{13-17}$ it is shown that several gravitating structures like stars, spiral galaxies, elliptical galaxies and clusters of galaxies can be self-consistently described without dark matter. There are other MONDian theories, for example, by introducing several scalar, vector and tensor fields, Bekenstein ${ }^{18}$ rewrote the MOND model of Milgrom in a covariant formalism (the TeVeS model). There is the Einstein-aether theory admitting a preferred reference frame and broken local Lorentz invariance. ${ }^{19}$

The accurate measurement of the speed of gravitational waves (GW) compared to the speed of light in 2017 ruled out modified gravity theories, termed "dark matter emulators", which dispense with the need for dark matter by making ordinary matter couple to a different metric from that of GW. These models have the property that, in the extreme weak field regime relevant to cosmology, gravitational waves propagate on different geodesics from those followed by photons and neutrinos. Therefore, the differential Shapiro delay between GWs and photons/neutrinos is due to the gravitational potential of only the dark matter. Some examples of these Dark Matter (DM) emulator theories include Bekenstein's TeVeS theory ${ }^{18}$ and Moffat's ScalarTensorVector gravity theory. ${ }^{20}$ It is important to understand that dark matter emulators constitute a special class of modified gravity theories which attempt to dispense with dark matter. Many modifications of gravity do not fall within this class, ${ }^{21}$ including Milgrom's bi-metric formulation of MOND, ${ }^{22}$ nonlocal MOND, ${ }^{23-25}$ such as superfluid dark matter, ${ }^{26}$ or dipolar dark matter. ${ }^{27}$ Nor does it apply to certain types of Einstein-Aether theories ${ }^{28,29}$ whose vector kinetic terms are properly chosen. Therefore, other kinds of modified gravity theories which dispense with the need for dark matter and are still viable. ${ }^{21}$ Some recent studies on MOND, $f(R)$ gravity, clustering of galaxies, and large distance modification of the Newtonian potential. ${ }^{30-34}$

In this section we shall review the main ingredients of Finsler geometry; ${ }^{35-45}$ present a solution of the vacuum field equation in Finsler gravity, in the weak field approximation, ${ }^{46,47}$ and show how it reproduces the main results of MOND. The solution depends on the rotational velocity of the galaxy consistent with the relationship between the Tully-Fisher relation ${ }^{48}$ and MOND. We finalize by showing how this Finsler gravity solution leads to a metric that is diffeomorphic (but not isometric) to the Hilbert-Schwarzschild metric. The most salient feature is that the metric is not asymptotically flat due to the infrared cutoff of the areal radial function, and resulting from imposing different boundary conditions for the metric at $r=\infty$ than in the Hilbert-Schwarzschild metric case. 
We shall begin with a very brief discussion of Finsler geometry ${ }^{35-45}$ before discussing the gravitational vacuum field equations. Finsler geometry is based on a non-negative real function $F\left(x, y=\frac{d x}{d \tau}\right)$, obeying $F(x, \lambda y)=\lambda F(x, y)$, and defined on the tangent bundle TM represented by the coordinates $x \equiv x^{0}, x^{1}, x^{2}, \cdots x^{n} ; y \equiv \frac{d x^{0}}{d \tau}, \frac{d x^{1}}{d \tau}, \cdots \frac{d x^{n}}{d \tau}$. The fundamental metric tensor is given as

$$
g_{\mu v}(x, y) \equiv \frac{1}{2} \frac{\partial^{2} F^{2}}{\partial y^{\mu} \partial y^{v}}
$$

The arc-length is

$L=\int F\left(x^{0}, x^{1}, \cdots x^{n} ; y^{0}, y^{1}, \cdots y^{n}\right) d \tau=\int F\left(x^{0}, x^{1}, \cdots x^{n} ; \frac{d x^{0}}{d \tau}, \frac{d x^{1}}{d \tau} \cdots \frac{d x^{n}}{d \tau}\right) d \tau$

The Cartan tensor (which measures the deviation from a Riemannian manifold) is given by the third derivative

$$
C_{\mu v \sigma}(x, y)=\frac{1}{4} \frac{\partial^{3} F^{2}}{\partial y^{\mu} \partial y^{v} \partial y^{\sigma}}
$$

If $C_{\mu \nu \sigma}(x, y)=0$ everywhere in the tangent space, the Finsler space becomes a metric space with $g_{\mu v}(x)$ independent on the tangent space coordinates $y$ (velocities). The geodesic equation on a Finsler manifold is given by

$$
\frac{d^{2} x^{\mu}}{d \tau^{2}}+2 G^{\mu}=0
$$

where the geodesic spray coefficients $G^{\mu}$ (dropping the $x, y$ dependence for convenience) are given by

$$
G^{\mu}=\frac{1}{4} g^{\mu \nu}\left(y^{\sigma} \frac{\partial^{2} F^{2}}{\partial x^{v} \partial y^{\sigma}}-\frac{\partial F^{2}}{\partial x^{v}}\right)
$$

The corresponding nonlinear connection $N_{v}^{\mu}(x, y)$ associated to the geodesic spray coefficients is defined by

$$
2 G^{\mu}(x, y)=N_{v}^{\mu}(x, y) y^{v}=\frac{1}{2} g^{\mu v}\left(y^{\sigma} \frac{\partial^{2} F^{2}}{\partial x^{v} \partial y^{\sigma}}-\frac{\partial F^{2}}{\partial x^{v}}\right)
$$

The nonlinear connection allows decomposing the tangent space to the tangent bundle $T_{(x, y)} T M$ at the point $(x, y)$ into a vertical space spanned by $\frac{\partial}{\partial y^{\mu}}$, and a horizontal space spanned by the socalled elongated derivatives $\frac{\delta}{\delta x^{\mu}} \equiv \frac{\partial}{\partial x^{\mu}}-N_{\mu}^{v} \frac{\partial}{\partial y^{\nu}}$. The nonlinear curvature derived from $N_{v}^{\mu}$ is

$$
R_{v \sigma}^{\mu} \equiv \frac{\delta N_{v}^{\mu}}{\delta x^{\sigma}}-\frac{\delta N_{\sigma}^{\mu}}{\delta x^{v}}, \frac{\delta}{\delta x^{\mu}} \equiv \frac{\partial}{\partial x^{\mu}}-N_{\mu}^{v} \frac{\partial}{\partial y^{v}}
$$

Regarding the tangent bundle $T_{M}$ as an $2(n+1)$-dim manifold of its own, one can construct linear covariant derivatives which are compatible with the structure induced by the nonlinear connection and which preserves the horizontal-vertical split of the tangent bundle $T M$ with basis $\frac{\delta}{\delta x^{\mu}}, \frac{\partial}{\partial y^{\mu}}$.
The nonlinear connection is unique, however the linear connections are not, and many different choices are possible. ${ }^{38-40}$ For example, the horizontal part of a torsionless linear connection is given in terms of the elongated derivatives $\delta / \delta x^{\mu}$ as

$$
\Gamma_{v \sigma}^{\mu}(x, y)=\frac{1}{2} g^{\mu \lambda}(x, y)\left(\delta_{v} g_{\sigma \lambda}(x, y)+\delta_{\sigma} g_{v \lambda}(x, y)-\delta_{\lambda} g_{v \sigma}(x, y)\right)
$$

and the horizontal part of the curvature (dropping $x, y$ ) is

$$
R_{v \sigma \lambda}^{\mu}=\delta_{\sigma} \Gamma_{v \lambda}^{\mu}-\delta_{\lambda} \Gamma_{v \sigma}^{\mu}+\Gamma_{\xi \sigma}^{\mu} \Gamma_{v \lambda}^{\xi}-\Gamma_{\xi \lambda}^{\mu} \Gamma_{v \sigma}^{\xi}-C_{v \xi}^{\mu} R_{\sigma \lambda}^{\xi}
$$

In Finsler geometry there is a geometrical invariant (under coordinate transformations) that only depends on the Finsler structure $F(x, y)$ and is insensitive to the choices of the linear connection. It is the Ricci scalar defined in terms of the geodesic spray coefficients as

$$
R \equiv R_{\mu}^{\mu}=\frac{1}{F^{2}}\left(2 \frac{\partial G^{\mu}}{\partial x^{\mu}}-y^{\lambda} \frac{\partial^{2} G^{\mu}}{\partial x^{\lambda} \partial y^{\mu}}+2 G^{\lambda} \frac{\partial^{2} G^{\mu}}{\partial y^{\lambda} \partial y^{\mu}}-\frac{\partial G^{\mu}}{\partial y^{\lambda}} \frac{\partial G^{\lambda}}{\partial y^{\mu}}\right)
$$

With these geometrical ingredients the analog of the Newtonian limit in Finsler spaces based on the notion of "locally Minkowski" spacetime. ${ }^{46,47}$ A Finsler spacetime is "locally Minkowski" if there is a coordinate system $x$ with induced tangent space coordinates $y$, such that $F$ only depends on $y$, but not on $x$. A "locally Minkowski" spacetime is a solution of the Finslerian vacuum field equations. ${ }^{41-45} \mathrm{X}$ Li et al. ${ }^{47}$ assumed a very small metric perturbation $h_{\mu v}(x, y)$ to the locally Minkowski one $\eta_{\mu v}(y)$

$$
g_{\mu v}(x, y)=\eta_{\mu v}(y)+h_{\mu v}(x, y),\left|h_{\mu v}(x, y)\right|<<1
$$

and found that to leading order in the post-Newtonian approximation the explicit form of the Finslerian line element which solves the vacuum field equations in a 4D Finsler spacetime is given by

$$
F^{2}(d \tau)^{2}=\left(1-\frac{2 G M}{R(r, v))}\right)(d t)^{2}-\left(1+\frac{2 G M}{R(r, v))}\right)(d R)^{2}-R^{2}(r, v)(d \Omega)^{2}
$$

where the radial coordinate in the locally Minkowski space-time of the galaxies is defined as $R(r, v) \equiv \sqrt{\eta_{i j}(v) x^{i} x^{j}}$. In this spherically symmetric case, the radial function is denoted by $R(r, v)$ (which must not be confused with the scalar curvature $R$ ), and now it depends on both $r$, and the velocity $v$, due to functional dependence of $g_{\mu \nu}(x, y)$ on both coordinates and velocities.

Li et al. ${ }^{46,47}$ have shown that Finsler gravity reduces to MOND if the spatial components of the locally Minkowski metric of galaxies is of the form

$$
\begin{aligned}
& \left.\eta_{00}=1, \eta_{i j}(y)=\delta_{i j}\left(1-\left(\frac{G M a_{o}\left(y^{0}\right)^{4}}{\left(\delta_{m n} y^{m} y^{n}\right)^{2}}\right)\right)^{2}\right)=\delta_{i j}\left(1-\left(\frac{G M a_{o}}{v^{4}}\right)^{2}\right) \\
& v^{i}=\frac{d x^{i}}{d x^{0}}=\frac{\left(d x^{i} / d \tau\right)}{\left(d x^{0} / d \tau\right)}=\frac{y^{i}}{y^{0}}
\end{aligned}
$$

where $a_{o}=1.2 \times 10^{-10} \mathrm{~m} / \mathrm{s}^{2}$ is the acceleration constant of MOND,, 5 and which is of the order of $\frac{c^{2}}{R_{H}}$, where $R_{H}$ is the present-
day Hubble scale. 
In this particular case the radial coordinate in the locally Minkowski space-time of the galaxies becomes

$R(r, v) \equiv \sqrt{\eta_{i j}(v) x^{i} x^{j}}=r f(v), f(v) \equiv \sqrt{1-\left(\frac{G M a_{o}}{v^{4}}\right)^{2}}, i, j=1,2,3$.

and the modified Newtonian equations of motion associated with the Finslerian line element (2.12) are given by

$\frac{G M}{R^{2}}=\frac{v^{2}}{R} \Rightarrow \frac{G M}{r^{2} f^{2}(v)}=\frac{v^{2}}{r f(v)} \Rightarrow \frac{G M}{r^{2}}=\frac{v^{2}}{r} f(v)=\frac{v^{2}}{r} \sqrt{1-\left(\frac{G M a_{o}}{v^{4}}\right)^{2}}$

One may recover the MONDian behavior from eq-(2.16) if $v(r)$ satisfies the following relation

$$
\frac{G M a_{o}}{v^{4}(r)}=\frac{1}{\sqrt{1+\left(\frac{v^{2}(r)}{r a_{o}}\right)^{2}}}
$$

upon inserting (2.17) into the last term of eq-(2.16) it allows to rewrite the scaling factor $f(v)$ in terms of $v$ and $r$, leading finally to the desired result of MOND

$$
\frac{G M}{r^{2}}=\frac{v^{2}}{r} f(v)=\frac{v^{2}}{r} \frac{\left(\frac{v^{2}}{r a_{o}}\right)}{\sqrt{1+\left(\frac{v^{2}}{r a_{o}}\right)^{2}}}=\frac{v^{2}}{r} \mu\left(\frac{v^{2}}{r a_{o}}\right)
$$

where

$$
\mu(x) \equiv \frac{x}{\sqrt{1+x^{2}}}, x \equiv \frac{v^{2}}{r a_{o}}
$$
learns

is the interpolating function in MOND. From eqs- $(2.18,2.19)$ one

$$
x<<1, \mu(x) \sim x ; x>>1, \mu(x) \sim 1
$$

The deep-MOND regime is characterized by $x<<1, \mu(x) \sim x$, such that

$$
r \rightarrow \infty, R(r) \rightarrow \frac{\sqrt{G M a_{o}}}{a_{o}}, v^{4}(r) \rightarrow G M a_{o}, f(v) \rightarrow 0
$$

and one recovers the Tully-Fisher relation $G M a_{o} \sim v^{4}$.

Introducing the following definitions in the deep infrared

$$
R_{c} \equiv R(r \rightarrow \infty), v_{c} \equiv v(r \rightarrow \infty)
$$

one can then rewrite (2.20b) in the following more familiar form

$$
\frac{G M}{R_{c}^{2}}=\frac{v_{c}^{2}}{R_{c}}=a_{o} \simeq \frac{c^{2}}{R_{H}}
$$

Given that $2 G M / c^{2} \equiv R_{S}$ is the definition of the Schwarzschild radius associated to a point mass $M$ gravitational source, from eq(2.21b) one arrives at the following scaling relations involving the Hubble radius $R_{H}, R_{S}$ and $R_{c}$

$$
\frac{1}{2} \frac{R_{S}}{R_{H}}=\left(\frac{v_{c}}{c}\right)^{4}, \frac{1}{2} R_{S} R_{H}=\left(R_{c}\right)^{2}
$$

Below we shall see the importance of these scaling relations (2.22) within the context of Black Hole Cosmology ${ }^{49-53}$ and Born's Reciprocal Relativity Theory. ${ }^{54-56}$

Given a galaxy of size $L_{g}$, from eq-(2.17) one can solve for $v=v(r)$ and obtain the rotational velocities for point test masses in the region $r \geq L_{g}$

$$
v^{4}(r)=\frac{\left(\frac{G M}{r}\right)^{2}+\sqrt{\left(\frac{G M}{r}\right)^{4}+4\left(G M a_{o}\right)^{2}}}{2}, r \geq L_{g}
$$

Eq-(2.23) is equivalent to the following expression

$$
r^{2}(v)=\frac{\left(v^{4} / a_{o}^{2}\right)}{\left(\frac{v^{4}}{G M a_{o}}\right)^{2}-1}, r \geq L_{g}
$$

and

$$
\frac{v^{2}}{r}=\frac{v^{4}}{G M} \sqrt{1-\frac{a_{o}^{2}}{\left(\frac{v^{4}}{G M}\right)^{2}}} \Leftrightarrow \frac{v^{4}}{G M}=a_{o} \sqrt{1+\frac{\left(v^{2} / r\right)^{2}}{a_{o}^{2}}}, r \geq L_{g}
$$

To sum up, given the range of scales

$$
R_{S}<R\left(r=L_{g}, v\left(L_{g}\right)\right) \simeq L_{g}<R(r, v(r))<R_{c}<R_{H}
$$

in the region $R(r, v(r)) \geq L_{g}$ one has $\frac{G M}{R^{2}}=\frac{v^{2}}{R}$ leading then to the rotational velocities associated to MONDian dynamics. In the interior region of the galaxy, ordinary Newtonian gravity is assumed to be valid, and for spherical symmetric mass distributions one has

$$
\frac{G M(r)}{r^{2}}=\frac{v^{2}}{r}, r<L_{g}
$$

which just follows from Gauss theorem when the mass enclosed $M(r) \mathrm{s}$ inside the spherical region of radius $r<L_{g}$ is given by $M(r)=\int_{0}^{r} \rho\left(r^{\prime}\right) 4 \pi r^{\prime 2} d r^{\prime}$.

Having gone through this Finsler geometric tour underlying MONDian dynamics, we can finally relate the results of this section with the previous one by noticing that in the regime $(2 G M / R)<<1$, a Taylor expansion yields

$$
\left(1-\frac{2 G M}{R}\right)^{-1} \sim\left(1+\frac{2 G M}{R}\right)
$$

and the Finslerian line element (2.12) in this regime reduces to

$F^{2}(d \tau)^{2}=\left(1-\frac{2 G M}{R(r, v)}\right)(d t)^{2}-\left(1-\frac{2 G M}{R(r, v)}\right)^{-1}(d R)^{2}-R(r, v)^{2}(d \Omega)^{2}$

leading then to a metric which is diffeomorphic (but not isometric) to the Hilbert-Schwarzchild one (after inserting the functional relation $v=v(r)$ given explicitly by eq-(2.23)) into the areal radial function $R(r, v)=R(r, v(r))$. In fact, the metric (2.29) is a solution to the vacuum Einstein field equations in a 4D spacetime for any functional form $v=v(r)$ with the provision that $R(-r, v(-r))=-R(r, v(r))$ as shown explicitly in the appendix a Lorentzian signature is used.

The particular form of $v(r)$ in eq-(2.23) was dictated to us by the empirical astronomical observations. Furthermore, from eq-(2.15) one learns that under the transformations

$$
r \rightarrow-r, M \rightarrow-M, a_{o} \rightarrow-a_{o} \Rightarrow R(r, v) \rightarrow-R(r, v)
$$

The areal radial function changes sign as it should be in order for the metric (2.29) to remain invariant. 
Despite that the metric (2.29) is diffeomorphic (but not isometric) to the Hilbert-Schwarzschild one, it is not asymptotically flat. The Kretschmann invariant $R_{\mu v \sigma \lambda} R^{\mu v \sigma \lambda} \sim\left(\frac{2 G M}{R^{3}(r, v(r))}\right)^{2} \neq 0$ is non-vanishing at $r=\infty$ due to the fact that $R(r=\infty)=R_{c}=\sqrt{G M a_{o}} / a_{o} \not \infty$ as shown in eq-(2.20b). In the limit that $a_{o}=0$, the areal radial function becomes the trivial one $R=r$, and one recovers the asymptotically flat Hilbert-Schwarzschild metric associated with ordinary Newtonian mechanics (in the weak field and slow moving bodies limit).

In a nutshell, simply by rewriting $\frac{G M}{R^{2}}$ as $\frac{G M_{e f f}(r)}{r^{2}}$, in terms of an effective mass $M_{\text {eff }}(r)$ enclosed in a spherical region of radius $r$, it leads to the relation $M_{\text {eff }}(r)=M / f^{2}(v(r)) \geq M$, since $f(v(r)) \leq 1$ , and such that the enhanced value of the "effective" mass $M_{\text {eff }}(r)$ compared to $M$ would seem as if non-luminous "dark matter" were present in the galaxies.

\section{Concluding remarks : scale invariance and born's reciprocal relativity theory}

By simple inspection one can verify that eqs-(2.15-2.26) are scale invariant under

$$
t \rightarrow \lambda t, r \rightarrow \lambda r, R(r, v) \rightarrow \lambda R(r, v), M \rightarrow \lambda M, a_{o} \rightarrow \lambda^{-1} a_{o}, v \rightarrow v, c \rightarrow c
$$

with $\lambda=$ constant and which implies a flat rotation curve. Note the anomalous scaling of the macroscopic galactic mass $M \rightarrow \lambda M$ as compared to the scaling $m \rightarrow \lambda^{-1} m$ of a fundamental particle (consistent with the scaling of the Compton wavelength $\hbar / m c$ ). Under these scalings (3.1) $f(v)$ given by eq-(2.15) is invariant and the metric (2.29) scales $(d s)^{2} \rightarrow \lambda^{2}(d s)^{2}$ as it occurs in Weyl's geometry under conformal transformations.

Another similar scaling occurs in Born's Reciprocal Relativity theory. It was shown that ${ }^{54-56}$ how one can implement a maximal proper force principle within the context of Born's Reciprocal Relativity theory, Mach's principle and Black-Hole Cosmology ${ }^{54-57}$ by setting the following proper forces to be equal to the maximal proper force value b ( "b" stands for Born)

$$
M_{U}\left(\frac{c^{2}}{R_{H}}\right)=m_{P}\left(\frac{c^{2}}{L_{P}}\right)=b
$$

where $M_{U}$ is the Universe's total mass inside the presentday Hubble radius $R_{H} ; m_{P}, L_{P}$ are the Planck mass, and length, respectively. What (3.2) indicates is that the observed Universe's total mass $M_{U}$ coincides with the product of the maximal proper force times the Hubble horizon scale (an infrared cutoff), and which in turn, is the black hole horizon radius corresponding to a Universe-mass black hole. The Planck mass is the product of the maximal proper force times the Planck scale (ultraviolet cutoff), and which in turn, is the black hole horizon radius corresponding to a Planck-mass black hole. And so forth, namely a black hole's mass $M$ coincides with the product of the maximal proper force $\mathrm{b}$ with its black hole horizon radius $R_{h}$.

Eq-(3.2) is also invariant under the scalings (3.1). Milgrom ${ }^{5,6}$ long ago pointed out the importance of scale invariance for MOND phenomenology, it is still impressive how much of that phenomenology, (i.e. flat rotation curves and baryonic Tully Fisher) is a consequence of scale invariance alone.

The recent new hypothesis ${ }^{58}$ is that this scale invariance is due to the dark matter undergoing a second order phase transition in the region normally associated with MONDian behavior. It is based on the idea that dark matter has a super-fluid phase ${ }^{59}$ which, if successful, would explain the flattening of the rotation curves, the Tully-Fisher ${ }^{48}$ and acceleration relations and the relation between $a_{o} \sim c^{2} / R_{H}$.

In this work we do not have to recur to these hypothesis. ${ }^{58}$ Eqs-
$(2.20,2.21)$ lead to these acceleration relations. A proposal that advocates the fall of dark matter can be found in. ${ }^{60,61}$ Scale invariance is assumed in the empty regions of space. The Weyl gauge field $A_{\mu}$ of dilatations contributes to modifications of the Christoffel connection leading then to repulsive corrections to the geodesic equations. We have not invoked the role of Quantum Gravity in this work nor what are the asymptotic symmetries (if any).

What we find remarkable is how powerful is the diffeomorphism symmetry of Einstein's vacuum field equations to account for the Finsler gravity solution described here, and which is able to model MOND by simply replacing the radial coordinate $r$ with the areal radial function $R(r, v(r))$. The key relation in eq- $(2.21 \mathrm{~b})$ could be interpreted as a balance equation between an inward acceleration due to MOND, and an outward acceleration due to the accelerated expansion of the Universe (since the observed cosmological constant $\Lambda=3 / R_{H}^{2}$ ). For this reason, it is warranted to find solutions of Finsler gravity which incorporate the cosmological constant, and see whether or not they can be recast in terms of diffeomorphic (but not isometric) solutions to the de Sitter-Schwarzschild metric.

\section{Appendix A: Schwarzschild-like solutions in D $>3$}

In this Appendix we verify Birkhoff's theorem by following closely the calculations of the static spherically symmetric vacuum solutions to Einstein's equations in any dimension $D>3$. Let us start with the line element with the Lorentzian signature $(-,+,+,+, \ldots .,+)$

$$
d s^{2}=-e^{\mu(r)}(d t)^{2}+e^{v(r)}(d r)^{2}+R^{2}(r) \tilde{g}_{i j} d \xi^{i} d \xi^{j} .
$$

where the areal radial function $\rho(r)$ is now denoted by $R(r)$ and which must not be confused with the scalar curvature $R$. Here, the metric $\tilde{g}_{i j}$ corresponds to a homogeneous space and $i, j=3,4, \ldots, D-2$ and the temporal and radial indices are denoted by 1,2 respectively. In our text we denoted the temporal index by 0 . The only non-vanishing Christoffel symbols are given in terms of the following partial derivatives with respect to the $r$ variable and denoted with a prime

$$
\begin{aligned}
& \Gamma_{21}^{1}=\frac{1}{2} \mu^{\prime}, \quad \Gamma_{22}^{2}=\frac{1}{2} v^{\prime}, \quad \Gamma_{11}^{2}=\frac{1}{2} \mu^{\prime} e^{\mu-v}, \\
& \Gamma_{i j}^{2}=-e^{-v} R R^{\prime} \tilde{g}_{i j}, \quad \Gamma_{2 j}^{i}=\frac{R^{\prime}}{R} \delta_{j}^{i}, \quad \Gamma_{j k}^{i}=\tilde{\Gamma}_{j k}^{i},
\end{aligned}
$$


and the only nonvanishing Riemann tensor are

$$
\begin{aligned}
& \mathcal{R}_{212}^{1}=-\frac{1}{2} \mu^{\prime \prime}-\frac{1}{4} \mu^{\prime 2}+\frac{1}{4} v^{\prime} \mu^{\prime}, \\
& \mathcal{R}_{121}^{2}=e^{\mu-v}\left(\frac{1}{2} \mu^{\prime \prime}+\frac{1}{4} \mu^{\prime 2}-\frac{1}{4} v^{\prime} \mu^{\prime}\right), \\
& \mathcal{R}_{i 1 j}^{1}=-\frac{1}{2} \mu^{\prime} e^{-v} R R^{\prime} \tilde{g}_{i j}, \\
& \mathcal{R}_{j k l}^{i}=\tilde{R}_{j k l}^{i}-R^{\prime 2} e^{-v}\left(\frac{1}{2} v^{\prime} R R^{\prime}-R R^{\prime \prime}\right) \tilde{g}_{i j}, \\
& \left.\tilde{g}_{j l}-\delta_{l}^{i} \tilde{g}_{j k}\right) .
\end{aligned}
$$

The vacuum field equations are

$$
\begin{aligned}
& \mathcal{R}_{11}=e^{\mu-v}\left(\frac{1}{2} \mu^{\prime \prime}+\frac{1}{4} \mu^{\prime 2}-\frac{1}{4} \mu^{\prime} v^{\prime}+\frac{(D-2)}{2} \mu^{\prime} \frac{R^{\prime}}{R}\right)=0, \\
& \mathcal{R}_{22}=-\frac{1}{2} \mu^{\prime \prime}-\frac{1}{4} \mu^{\prime 2}+\frac{1}{4} \mu^{\prime} v^{\prime}+(D-2)\left(\frac{1}{2} v^{\prime} \frac{R^{\prime}}{R}-\frac{R^{\prime \prime}}{R}\right)=0,
\end{aligned}
$$

and

$$
\mathcal{R}_{i j}=e^{-v} R^{2}\left(\frac{1}{2}\left(v^{\prime}-\mu^{\prime}\right) R R^{\prime}-R R^{\prime \prime}-(D-3) R^{\prime 2}\right) \tilde{g}_{i j}+k R^{2}(D-3) \tilde{g}_{i j}=0,
$$

where $k= \pm 1(A .6)$, depending if $\tilde{g}_{i j}$ refers to positive or negative curvature. From the combination $e^{-\mu+v} R_{11}+R_{22}=0$ we get

$$
\mu^{\prime}+v^{\prime}=\frac{2 R^{\prime \prime}}{R^{\prime}} .
$$

The solution of this equation is

$$
\mu+v=\ln R^{\prime 2}+C,
$$

where $\mathrm{C}$ is an integration constant that one sets to zero if one wishes to recover the flat Minkowski spacetime metric in spherical coordinates in the asymptotic region $r \rightarrow \infty$.

Substituting (A.7) into the equation (A.6) we find

$$
e^{-v}\left(v^{\prime} R R^{\prime}-2 R R^{\prime \prime}-(D-3) R^{\prime 2}\right)=-k(D-3)
$$

or

$$
\gamma^{\prime} R R^{\prime}+2 \gamma R R^{\prime \prime}+(D-3) \gamma R^{\prime 2}=k(D-3),
$$

\section{Where}

$$
\gamma=e^{-v} \text {. }
$$

The solution of (A.10) for an ordinary D-dim spacetime ( one temporal dimension ) corresponding to a D-2 -dim sphere for the homogeneous space can be written as

$$
\begin{aligned}
& \gamma=\left(1-\frac{16 \pi G_{D} M}{(D-2) \Omega_{D-2} R^{D-3}}\right)\left(\frac{d R}{d r}\right)^{-2} \Rightarrow \\
& g_{r r}=e^{v}=\left(1-\frac{16 \pi G_{D} M}{(D-2) \Omega_{D-2} R^{D-3}}\right)^{-1}\left(\frac{d R}{d r}\right)^{2} .
\end{aligned}
$$

where $\Omega_{D-2}$ is the appropriate solid angle in $D-2$-dim and $G_{D}$ is the D-dim gravitational constant whose units are (length $)^{D-2}$ . Thus $G_{D} M$ has a unit of (length) ${ }^{D-3}$ as it should. When $D=4$ as a result that the 2-dim solid angle is $\Omega_{2}=4 \pi$ one recovers from eq-
(A.12) the 4-dim Schwarzchild solution. The solution in eq-(A.12) is consistent with Gauss law and Poisson's equation in D-1 spatial dimensions obtained in the Newtonian limit.

For the most general case of the D-2-dim homogeneous space we should write

$$
-v=\ln \left(k-\frac{\beta_{D} G_{D} M}{R^{D-3}}\right)-2 \ln R^{\prime}
$$

$\beta_{D}$ is a constant equal to $16 \pi /(D-2) \Omega_{D-2}$, where $\Omega_{D-2}$ is the solid angle in the $D-2$ transverse dimensions to $r, t$ and is given by $2 \pi^{(D-1) / 2} / \Gamma[(D-1) / 2]$.

Thus, according to (A.8) we get

$$
\mu=\ln \left(k-\frac{\beta_{D} G_{D} M}{R^{D-3}}\right)+\text { constant } .
$$

we can set the constant to zero, and this means the line element (A.1) can be written as

$$
\begin{aligned}
& d s^{2}=-\left(k-\frac{\beta_{D} G_{D} M}{R^{D-3}}\right)(d t)^{2}+\frac{(d R / d r)^{2}}{\left(k-\frac{\beta_{D} G_{D} M}{R^{D-3}}\right)}(d r)^{2}+R^{2}(r) \tilde{g}_{i j} d \xi^{i} d \xi^{j}= \\
& -\left(k-\frac{\beta_{D} G_{D} M}{R^{D-3}}\right)(d t)^{2}+\frac{1}{\left(k-\frac{\beta_{D} G_{D} M}{R^{D-3}}\right)}(d R)^{2}+R^{2}(r) \tilde{g}_{i j} d \xi^{i} d \xi^{j}
\end{aligned}
$$

One can verify, that the equations (A.4)-(A.6), leading to eqs(A.9)-(A.10), do not determine the form $R(r)$. It is also interesting to observe that the only effect of the homogeneous metric $\tilde{g}_{i j}$ is reflected in the $k= \pm 1$ parameter, associated with a positive (negative) constant scalar curvature of the homogeneous $D-2$-dim space. $k=0$ corresponds to a spatially flat $D-2$-dim section. The metric solution in eq-(1.2) is associated to a different signature than the one chosen in this Appendix, and corresponds to $D=4$ and $k=1 .{ }^{62-73}$

\section{Acknowledgments}

We are indebted to M. Bowers for assistance

\section{Conflicts of interest}

Authors declare there is no conflict of interest.

\section{References}

1. A Einstein. The Field Equations of Gravitation. Berlin: Sitzungsber Preuss Akad; 1915. p. 831.

2. K Schwarzschild. On the gravitational field of a mass point according to Einstein's theory. Berlin: Sitzungsber Preuss Akad; 1999. p.7.

3. C Castro. Novel Remarks on Point Mass Sources, Firewalls, Null Singularities and Gravitational Entropy. Foundations of Physics. 2016;46(1):14-27.

4. Carlos Castro. Asymptotic Safety in Quantum Gravity and Diffeomorphic Non-isometric Metric Solutions to the Schwarzschild Metric. Canadian Journal of Physics. 2018;96(1):90-97.

5. M Milgrom. A modification of the Newtonian dynamics as a possible alternative to the hidden mass hypothesis. Astrophysical Journal. 1983;270:365-370. 
6. P Kroupa, M Pawlowski, M Milgrom. The failures of the standard model of cosmology require a new paradigm. International Journal of Modern Physics D. 2012;21(14):1230003.

7. A Almheiri, D Marolf, J Polchinski, et al. Black Holes: Complementarity or Firewalls. Journal of High Energy Physics. 2013;62.

8. L Susskind, L Thorlacius, J Uglum. The Stretched Horizon and Black Hole Complementarity. Phys Rev D. 1993;48:3743.

9. F Klinkhamer. A new type of nonsingular black-hole solution in general relativity. Mod Phys Lett. 2014;29(19):1430018.

10. VC Rubin, N Thonnard, WK Ford. Extended rotation curves of highluminosity spiral galaxies. IV - Systematic dynamical properties, SA through SC. Astrophysical Journal Letters. 1978;225:107-111.

11. VC Rubin, WK Ford, N Thonnard. Rotational properties of $21 \mathrm{SC}$ galaxies with a large range of luminosities and radii, from NGC $4605 / \mathrm{R}=4 \mathrm{kpc}$ to UGC $2885 / \mathrm{R}=122 \mathrm{kpc} /$. Astrophysical Journal. 1980;238:471-487.

12. M Lopez Corredoira. Tests and problems of the standard model in Cosmology. arXiv: 1701.08720 .

13. S Capozziello, M De Laurentis. The dark matter problem from $f(R)$ gravity viewpoint. Annalen der Physik. 2012;524(9-10):545-578.

14. S Capozziello, M De Laurentis. Extended Theories of Gravity. Phys Rep. 2011;509:(4-5):167-321.

15. S Capozziello, P Jovanovic, V Borka Jovanovic et al. Addressing the missing matter problem in galaxies through a new fundamental gravitational radius. JCAP. 2017;06:044.

16. BG Sidharth, A Das. Anomaly of Dark Matter. arXiv: 1609.04246.

17. P Mannheim. Alternatives to Dark Matter and Dark Energy. Prog Part Nucl Phys. 2005;56(2):340.

18. J Bekenstein. Relativistic gravitation theory for the modified Newtonian dynamics paradigm. Physical Review D. 2004;70(8):083509.

19. T Zlosnik, P Ferreira, G Starkman. Modifying gravity with the aether: An alternative to dark matter. Phys Rev D. 2007;75:044017.

20. JW Moffat. Scalar-tensor-vector gravity theory. Journal of Cosmology and Astroparticle Physics. 2006;3:004.

21. S Boran, S Desai, E Kahya, et al. GW170817 Falsifies Dark Matter Emulators. Phys Rev D. 2018;97:041501.

22. M Milgrom. Bimetric MOND gravity. Phys Rev D. 2009;80:123536.

23. C Deffayet, G Esposito Farese, RP Woodard. Nonlocal metric formulations of modified Newtonian dynamics with sufficient lensing. Phys Rev D. 2011;84:124054.

24. C Deffayet, G Esposito Farese, RP Woodard. Field equations and cosmology for a class of nonlocal metric models of MOND. Phys Rev D. 2014;90:064038.

25. M Kim, MH Rahat, M Sayeb, et al. Determining cosmology for a nonlocal realization of MOND. Phys Rev D. 2016;94:104009.

26. L Berezhiani, J Khoury. Theory of dark matter superfluidity. Phys Rev D. 2015;92:103510.

27. L Blanchet, L Heisenberg. Dipolar dark matter as an effective field theory. Phys Rev D. 2017;96:083512.

28. RH Sanders. Hiding Lorentz invariance violation with MOND. Phys Rev D. 2011;84:084024.

29. L Blanchet, S Marsat. Modified gravity approach based on a preferred time foliation. Phys Rev D. 2011;84:044056.

30. S Capozziello, M Faizal, M Hameeda, et al. Clustering of galaxies with $\mathrm{f}(\mathrm{R})$ gravity. Monthly Notices of the Royal Academic Society. 2018;474(2):2430-2443.
31. M Hameeda, S Upadhyay, M Faizal, et al. Effects of cosmological constant on clustering of Galaxies. Monthly Notices of the Royal Academic Society. 2016;463:3699-3704.

32. S Upadhyay. Thermodynamics and galactic clustering with a modified gravitational potential. Phys Rev D. 2017;95:043008.

33. B Pourhassan, S Upadhyay, M Hameeda, et al. Clustering of galaxies with dynamical dark energy. Monthly Notices of the Royal Academic Society. 2017;468:3166.

34. M Hameeda, S Upadhyay, M Faizal, et al. Large distance modification of Newtonian potential and structure formation in universe. Physics of the Dark Universe. 2018;19:137-143.

35. P Finsler. Über Kurven und Flächen in allgemeinen Räumen. $\mathrm{PhD}$ thesis, Georg August University zu Göttingen, Germany. 1918

36. D Bao, S Chern, Z Shen, An Introduction to Riemann-Finsler Geometry. Graduate Texts in Mathematics. NewYork; Springer: 2000. p. 435.

37. R Miron, D Hrimiuc, H Shimada, et al. The Geometry of Hamilton and Lagrange Spaces. Netherlands: Springer; 2002. p. 338.

38. S Vacaru. On axiomatic formulation of gravity and matter field theories with MDRs and Finsler-Lagrange-Hamilton geometry on (co)tangent Lorentz bundles. arXiv: 1801.06444.

39. S Vacaru. Finsler-Lagrange Geometries and Standard Theories in Physics: New Methods in Einstein and String Gravity. arXiv: 0707.1524.

40. S Vacaru, P Stavrinos, E Gaburov, et al. Clifford and Riemann-Finsler Structures in Geometric Mechanics and Gravity. Differential Geometry - Dynamical Systems Monographs. 2003. p. 693.

41. C Pfeifer. The Finsler spacetime framework: backgrounds for physics beyond metric geometry. Ph.D thesis, University of Hamburg. 2013. p 151

42. C Pfeifer. From the Einstein-Hilbert action to an action principle for Finsler gravity. Diploma thesis, University of Hamburg. 2010. p. 91.

43. C Pfeifer, MNR Wohlfarth. Finsler geometric extension of Einstein gravity. Phys Rev D. 2012;85:064009.

44. S Rutz. A Finsler generalization of Einstein's vacuum field equations. General Relativity and Gravitation. 1993;25(11):1139-1158.

45. M Hohmann. Extensions of Lorentzian spacetime geometry: From Finsler to Cartan and vice versa. Phys Rev D. 2013;87:124034.

46. X Li, Z Chang. The spacetime structure of MOND with TullyFisher relation and Lorentz invariance violation. Chinese Physics $C$. 2013;37(12):123103

47. X Li, MH Li, HN Lin, et al. Finslerian MOND vs. observations of Bullet Cluster 1E0657-558. Mon Not Roy Astron Soc. 2013;428: 2939-2948.

48. RB Tully, JR Fisher. A new method of determining distances to galaxies. Astron Astrophys. 1977;54(3):661-673.

49. RPathria. The Universe as a BlackHole. Nature. 1972;240(5379):298-299.

50. IJ Goody. Chinese universes. Physics Today. 1972;25:7.

51. P Landsberg. Mass Scales and the Cosmological Coincidences. Annalen der Physik. 1984;496(2):88-92.

52. N Poplawski. Universe in a Black-Hole in Einstein-Cartan Gravity. Astrophys J. 2016;832(2):96.

53. Black Hole Cosmology

54. C Castro. Is Dark Matter and Black-Hole Cosmology an Effect of Born's Reciprocal Relativity Theory? Canadian Journal of Physics. 2018.

55. C Castro. On Dual Phase Space Relativity, the Machian Principle and Modified Newtonian Mechanics. Progress in Physics. 2005;1:20-30. 
56. C Castro. Solutions to the Gravitational Field Equations in Curved PhaseSpaces. Electronic Journal of Theoretical Physics. 2018;14(37):145-160

57. SK Blau, EI Guendelman, AH Guth. Dynamics of false-vacuum bubbles. Phys Rev D. 1987;35(6):1747.

58. S Alexander, L Smolin. The Equivalence Principle and the Emergence of Flat Rotation Curves. arXiv:1804.09573.

59. MP Silverman, RL Mallett. Dark matter as a cosmic Bose-Einstein condensate and possible superfluid. Gen Rel Grav. 2002;34(5):633-649.

60. A Maeder. Dynamical effects of the scale invariance of the empty space: The fall of dark matter. 2017;849(2):158.

61. A Maeder. An Alternative to the $\Lambda$ CDM Model: The Case of Scale Invariance. The Astrophysical Journal. 2017;834(2):194.

62. D Hilbert. Mathematische Probleme. Nachr Ges Wiss Gottingen Math Phys. 1917;K1:53.

63. H Weyl. Republication of: 3. The theory of gravitation. Ann Physik (Leipzig). 1917;54:117-145.

64. J Droste. Proc Ned Akad West Ser. 1917;A19:197.

65. C Fronsdal. Completion and Embedding of the Schwarzschild Solution. Phys Rev. 1959;116:778.
66. M Kruskal. Maximal Extension of Schwarzschild Metric. Phys Rev. 1960;119:1743.

67. G Szekers. On the singularities of a Riemannian manifold. Publ Mat Debreca. 1960;7:285.

68. AAkil, O Dahlsten, L Modesto. A Firepoint at the Black Hole Singularity. arXiv: 1805.04368

69. J Oort Bull. The force exerted by the stellar system in the direction perpendicular to the galactic plane and some related problems. Bulletin of the Astronomical Institutes of the Netherlands. 1932;6:249.

70. F Zwicky Helv. Die Rotverschiebung von extragalaktischen Nebeln. Helvetica Physica Acta. 1993;6:110-127.

71. M Milgrom. Scale Invariance at low accelerations (aka MOND) and the dynamical anomalies in the Universe. Progress in physics. 2017;65(6-8):1600046.

72. M Mordehai. MOND laws of galactic dynamics. Monthly Notices of the Royal Astronomical Society. 2014;437(3):2531-2541.

73. M Milgrom. Dynamics with a Nonstandard Inertia-Acceleration Relation: An Alternative to Dark Matter in Galactic Systems. Annals of Physics. 1994;229(2):384-415. 